\title{
An Unmanned Aerial Vehicle Cluster Network Cruise System for Monitor
}

\author{
Jirong Jiang ${ }^{1}$, Jinpeng $\mathrm{Tao}^{2}$, Guipeng $\mathrm{Xin}^{3}$ \\ ${ }^{1}$ Wuhan University of Technology, Wuhan, China \\ ${ }^{2}$ Wuhan University of Technology, Wuhan, China
}

\begin{abstract}
The existing maritime cruising system mainly uses manned motorboats to monitor the quality of coastal water and patrol and maintenance of the navigation -aiding facility, which has the problems of high energy consumption, small range of cruise for monitoring, insufficient information control and low visualization. In recent years, the application of UAS in the maritime field has alleviated the phenomenon above to some extent. A cluster-based unmanned network monitoring cruise system designed in this project uses the floating small UAV self-powered launching platform as a carrier, applys the idea of cluster, and combines the strong controllability of the multi-rotor UAV and the capability to carry customized modules, constituting a unmanned, visualized and normalized monitoring cruise network to realize the functions of maritime cruise, maintenance of navigational-aiding and monitoring the quality of coastal water.
\end{abstract}

\section{Introduction}

With the development of the marine economy, the rapid development of the inland waterway transport, the distribution pattern of water transport and the risk of water traffic undergone tremendous changes, the task of safeguarding national sovereignty and maritime rights has been more prominent and the task of water safety management, marine life rescue and marine environment prevention and control has been getting more and more burdensome for the maritime departments. On May 31, 2017, Qinhuangdao Maritime Board organized UAV cruise.

operations to accurately grasp the actual distribution of aquaculture areas under administration, ship navigation in coastal waters and on-site construction operations, and help the maritime departments to grasp the information of the traffic conditions of vessels in key areas under administration and navigation dynamics more quickly and help the marine department effectively enhance the capability of warning, decision-making, command and disposal of emergency at the same time in the search and rescue of lives at sea .

At present, all maritime boards have gradually adopted sea cruising craft, unmanned aerial vehicles and shorebased integrated three-dimensional cruising system to step up maritime cruising and enhance the safety of navigation of ships. However, the existing cruise systems have the following problems.

(1) High energy consumption. The existing maritime cruising system mainly uses manned motorboats and large UAV to monitor the quality of coastal water and patrol and maintenance of the navigation-aiding facility with the problems of low autonomy of the weather, extremely large consumption of fossil fuel and high cost of maintenance and operating. To Guangdong Maritime board, for example, the annual fuel costs are 431,000 yuan and 204,800 yuan, the airport fees, insurance and other costs are 3.334 million yuan and 3.18 million yuan, the average cruise cost per unit of sea is 55.86 yuan and 221.55 yuan.

(2) Small cruise monitoring range. The cruising radius of manned motor boats and unmanned aerial vehicles are often delineated by half of their maximum range,because UAV needs the support of mother ships and shore-based charging stations, their cruising range is often limited by their mother ship or shore-based charging stations ,thus their cruise effect cannot be maximized.

(3) Informationcan not be obtained in time. Traditional methods for monitoring the water quality are the artificial tedious steps of assay after the manual sampling, with the problems for samples of small number, small scope, poor timeliness and so on, resulting in the monitoring of the sea not be centralized, real-time and large-scale.

In response to the increasingly severe maritime security situation, and existing cruise system problems, there is an urgent need for a new cluster unmanned network monitoring cruise system with a wide range of cruise monitoring, sufficient energy supply and timely information feedback so as to improve the monitoring efficiency.

\section{Design principles}

\subsection{Design ideas}


We take designing a "cluster unmanned network monitoring cruise system" as the goal, first of all, design a capable of providing unmanned aerial floating platform, take the floating charging platform as the base and make reasonable arrangement in the offshore area to form a unmanned cluster network, leading to greatly extend of the UAVs cruise time and expanding of the cruising range of UAVs .By adding custom modules to unmanned aerial vehicles, realizing the unmanned monitoring of water quality, navigational aids, etc., and more timely information transmission can provide more accurate information on offshore maritime work.

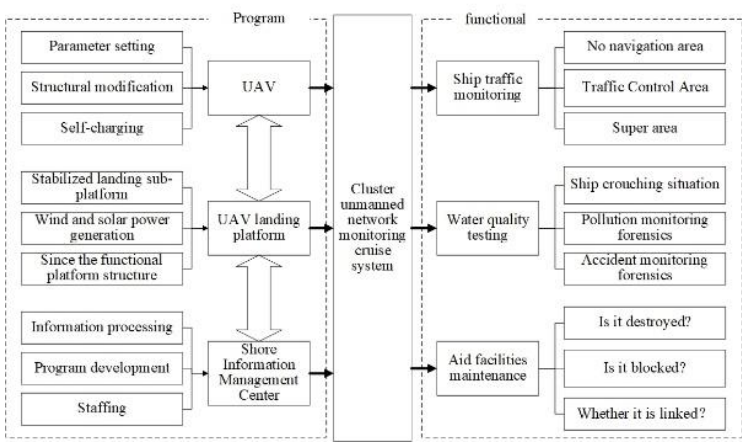

Figure 1. construction of UAV cluster network monitoring

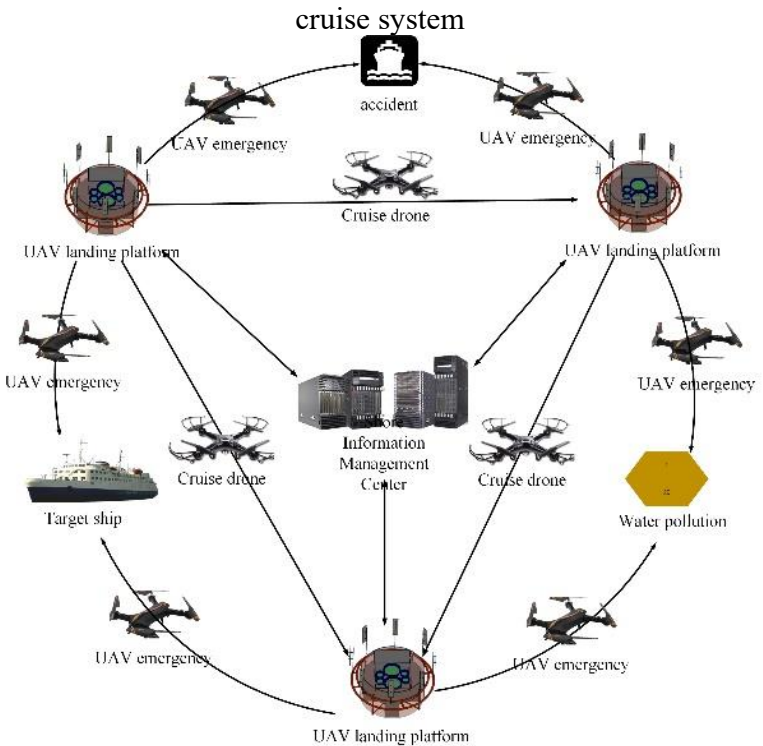

Figure 2. operation of UAV cluster network monitoring cruise system

\subsection{The design of the whole program}

The cruising UAV continuously collects video images of waterways in normal cruising and sends image information and self-status information to shore-based management center through the closer self-powered UAV landing platform. When the shore-based management center detects a ship accident, it sends an emergent order to the self-powered UAV landing platform which is closer to the accident site and the platform calls the emergent UAV to leave for the accidental waters to monitor and collect evidence, assisting search and rescue personnel in emergent handling of the accident, after that UAV continues to its scheduled conventional cruise. When there is a key ship entering the port and the maritime department needs to monitor it in real time, the shore-based management center send special monitoring instructions to the self-generating UAV Landing platform that closer to the ship route, the platform calls the emergent UAV to carry out follow-up monitoring of the whole relay, the realtime image information is fed back to the maritime regulatory authorities. Through the unmanned aerial vehicle equipped with maritime monitoring system, import the water image into the established neural network model to obtain the relevant data of the water body. When it is found that the water in the monitoring area is polluted, the system immediately sends an early warning to the shorebased staff, the relevant departments should immediately enter the emergent state and the shore-based management center shall call emergent UAV formation quickly activated and arrive in the area for on-site forensics and identification of sources of contamination. And feedback the type, degree, range and other information of the pollution timely.

\subsection{The design structure of small floating self- generating UAV landing platform}

The platform structure is composed of upper, middle and lower three layers as a whole, as shown in the figure, which is mainly composed of three modules of wind and solar power generating units, stable UAV landing platform, energy conversion module and etc.

The upper wind and solar power generation units include vertical axis wind power plants and solar power plants, and it has two vertical axis wind power plants. To ensure its stability, the bottom of it is connected to the bottom of the platform and go through the upper surface of the platform, symmetrically distributed on it, and the upper blade is used to capture wind power. The solar power generation device is mainly composed of three solar panels and a control device. Each solar panel is fixed by the pillar connection provided on the bottom of the landing platform and can be rotated and controlled around the axis of the contact point. The solar panel can automatically adjust the rotation angle through the photosensitive, maximizing the use of solar energy. In addition, the solar panel serves as the landing door of the landing platform, when the UAV needs charging from the power generation platform, the solar panel is sensed by the sensor and rotated to a position vertical to the upper surface to open the landing platform for the UAV.

The middle space, which is just below the solar panel door, is used to fix up the stable UAV landing and charging platform, including two cruise UAV landing and charging platforms and four emergent UAV landing and charging platforms, with the solar panel as the axis, arranged symmetrically, the specific distribution is shown as follow:

The lower space is the core component layer. This area has a sealed structure for storing energy storage, control circuit and information relay module to ensure the normal operation of the entire system. 


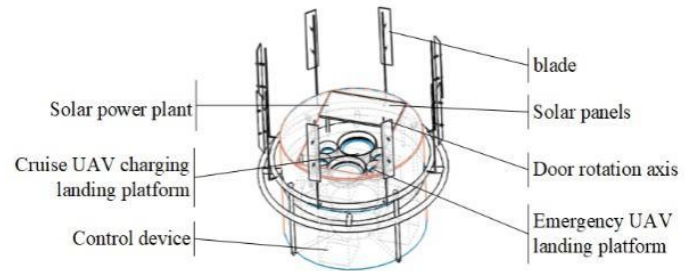

Figure 3. main structure diagram of the platform

The overall appearance of the platform is a cylindrical, with the bottom radius of 1.8 meters, height of 2 meters. In addition to the bottom part and the upper surface except by the three rotating solar panels outside the area, the platform is a closed compartment made of transparent materials, which can keep effectively off the wind and rain to protect the UAV.

\subsection{UAV conversion design}

Modified part mainly includes landing, charging device and protection if falling into the water of UAV in three parts.

\section{UAV landing structure}

According to the design of the stable UAV landing platform, caster is added to its four landing positions so that the UAV can slide down the circular slope of the platform within the allowable range and finally stop at the charging coil.

\section{UAV charging device}

In order to realize the wired charging of the UAV in the stable landing and charging platform, a wired charging device is specially designed at the bottom of the UAV. The wired charging device includes a positive charging annular groove, the size of the annular groove is coupled with the negative charging ring of the stable platform, and the negative charging contact can make contact with the positive electrode circular plate of the stable platform after the UAV lands to form a loop to start charging.

\section{UAV protection device}

UAV is expensive and often encounter stormy weather in actual cruise search and rescue process, UAV may fall into the water when landing on the platform or it breaks down. And UAV did not design watertight structure, so it will cause damage once it falls into the water. Therefore, we design the protective airbag at the bottom of the UAV, thus only the airbag on the bottom contacting with the water when the UAV falls into the water, then floats on the water waiting for manual recovery.

\section{5 design module for remote control}

UAV equipped with camera PTZ, water quality monitoring module, positioning module to help collect information of the status of navigation-aiding facility, images of water quality, its location or power and so on, and the information transforms long-distance transmission of signals to shorebased information management system through relay transmission from self-powered landing platform. Using computer to contact server, recept and run the remote monitoring software based on QT design processing, and analyses the data from the sensor on the self-powered landing platform. Then the shore-basing operator sends relevant instruction to control the operating of the selfpowered landing platform and UAV to realize the longdistance control and management of the system and bidirectional transmission of information.

\section{6 monitoring water quality and treatment of sudden cases of the ships for UAV}

With the development of technology and the improvement of concept of environmental protection, environmental monitoring technology for UAV is inevitably one of the mainstream trends in the development of environmental protection technologies. In recent two decades, China has built the world's largest and most expensive online pollution emission monitoring system, costing tens of billions of yuan. However, according to the current situation, this system is difficult to prevent fraud and waste disposal, which is precisely one of the most important reasons for the deterioration of air and water environment in China. In the monitoring work of water quality in ports, the maritime departments use the UAV remote sensing image supervising site to supervise the scene to process evidence quickly, interprets aerial infrared thermal images, use spectral telemetry environmental monitoring technology to extract pollutant characteristics, provide high-resolution photos and interpretation data, capture other environmental violations and forensics timely.

Based on realizing the UAV cluster cruise, the system immediately sends an early warning to shore-based staff when it detects the presence of water quality pollution in the monitored area by carrying a marine monitoring system for water quality on the UAV, and the relevant departments should immediately enter the emergent state and the shorebased management center shall call emergent UAV formation quickly activated and arrived in the area for onsite forensics and identification of sources of contamination. And feedback the type, degree, range and other information of the pollution timely, carry out corresponding emergent measures according to specific water pollution, delineate the need to isolate the contaminated areas, thereby effectively avoiding the spread of the pollution and expansion in the accident site.

The cruising UAV continuously collects video images of channel waters in normal cruising and sends image information and self-status information to shore-based management center through the closer self-powered UAV landing platform. When the shore-based management center detects a ship accident, it sends an emergent order to the self-powered UAV landing platform that is closer to the accident site. The platform calls the emergent UAV to leave for the accidental waters to monitor and collect evidence, assisting search and rescue personnel in emergent handling of the accident, then the UAV continues its default routine cruise. 
When there is a key ship entering the port and the maritime department needs to monitor it in real time, the shore-based management center send special monitoring instructions to the self-generating UAV Landing platform closer to the ship route, the platform calls the emergent UAV to carry out follow-up monitoring of the whole relay, and the real-time image information is fed back to the maritime regulatory authorities.

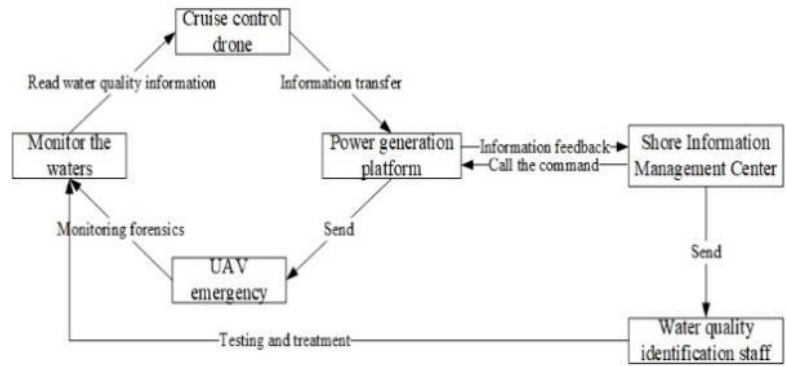

Figure 4. flow chart of emergency response to water pollution

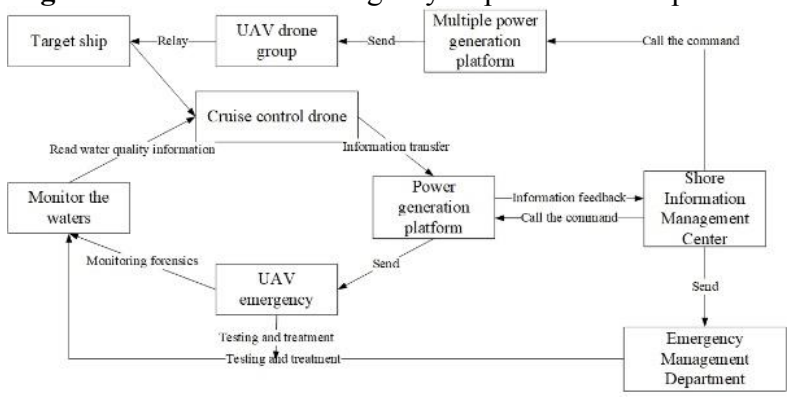

Figure 5. flow chart of emergency response

\section{7 comparative analysis}

As the platform is used in offshore waters, taking the various parameters of the Bohai Sea as an example, to calculate the capacity of the generation of solar-wind power generation platform. According to the monthly total solar radiation and the average wind speed in the Bohai Sea, and import the power consumption and power generation calculation formula, the calculated power generation and power consumption is compared as the Figure 6.

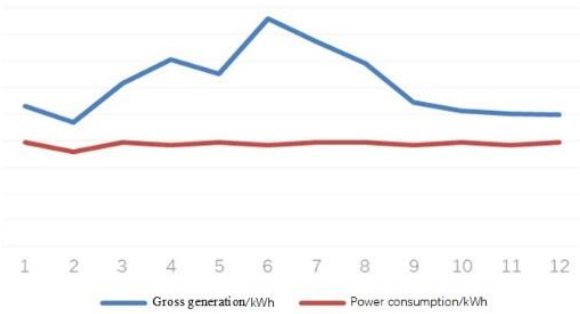

Figure 6. comparison chart of total power generation and power consumption

According to figure 8, the solar-wind power generation system can guarantee the power consumption of various equipment on the platform and provide enough power for the UAV lithium battery.

\section{3 innovation and application prospects}

\section{1 innovation}

1.The design of the small floating self-generating UAV landing platform. A multi-layer structure is designed to separate the stable UAV landing platform, power generation equipment and platform circuit components and to realize the full functionality of the platform on a limited area available. At the same time, the use of the platform effectively mitigated the short time of edurance of the multi-rotor UAV to impact its ability of cruising, making it possible for the medium-sized multi-rotor UAVs to be used in maritime cruising.

2. Integrated remote-control system. The information is relayed to the shore-basing information management system through relay transmission from the selfgeneration and landing platform. The remote monitoring software processes and analyzes the data from the sensors on the self-generation and landing platform to the shorebasing operators, the shore-basing operators can issue related commands to control the self-generation landing platforms and UAV to achieve remote control and management of the system and bidirectional transmission of information.

3. Monitoring program based on neural network model. Through the special surveillance system installed on the $\mathrm{UAV}$, the water image is imported into the established neural network model, and the relevant data of the water body can be obtained after the operation of image cutting, feature extraction and other operations. The suspicious accidental waters may also be dispatched to supervise by the strength of surveillance.

4. Construction of a new type of UAV cluster network monitoring cruise system. Taking the floating platform of small self-powered UAV landing as the node, the UAV's trajectory is planned, the UAV cluster monitoring network is constructed, the visual supervision of offshore vessels' traffic and the continuous monitoring of the normalization of navigation- aiding facilities and offshore water quality are realized.

\section{2 application prospects}

With the development of science and technology and the unmanned and intelligent trend of future maritime cruising, the idea of clustering UAVs can be realized in the offshore monitoring cruise after the design of self-powered UAV landing platform solves the problem of UAV energy supplement. Through the separation of different watersheds and the rational design of patrol schemes, UAV stealth enforcement will become a notable feature of future maritime regulatory technology and will play an prominent role in future maritime for cruise monitoring.

\section{References}

1. Li Huawei. Research And Realization for Multi-UAV Cooperative Mission Planning[D] Xi'an University of Electronic Science and Technology,2014.

2. Yu Yongjun. Research on Key Technologies for High Altitude and Long-flight-time UAV Information Fusion Autonomous Navigation [D] Nanjing University of Aeronautics and Astronautics,2011. 
3. Yu Sinan, Zhou Rui, Xia Jie, Che Jun. Decomposition and coverage of multi-UAV cooperative search area $[\mathrm{J}]$. Journal of Beijing University of Aeronautics and Astronautics,2015,41(01):167-173.

4. Liu Fan, Shi Qianqing, Fei Xianyun. Object-oriented Remote Sensing for the Variation of Sea Area Utilization [J]. Journal of Huaihai Institute of Technology (Natural Science Edition),2015,24(02):82-86.

5. Liu Fuhu, Ma Xiaoping. Research for improving the endurance performance of minitype electric-powered UAV [J]. Flight Dynamics,2010,28(05):13-15.

6. Anonymous. ONR Awards Boeing \$23 Million To Finish Free Electron Laser Design[J]. Defense Daily,2010,247(55).

7. Carlo Corradini,Lisa De Propris. Beyond local search: Bridging platforms and inter-sectoral technological integration[J]. Research Policy, 2016.

8. Pavo Ritala,Eelko Huizingh ,Argyro Almpanopoulou,Paul Wijbenga. Tensions in R\&amp;D networks: Implications for knowledge search and integration[J]. Technological Forecasting \&amp; Social Change, 2016.

9. Ma Minglu. Research on the Crucial Problems in Integration of Maritime Cruise Search and Rescue [J]. China Water Transport ,2016(7):36-38. 